\title{
Development and Error Analysis of 3D Laser Tracking Ball Bar
}

\author{
Ying-zhi Zhang ${ }^{1}$, Gong-chang Ren ${ }^{2}$, and Hai-tao $\mathrm{Li}^{2}$ \\ ${ }^{1}$ Shaanxi University of Science and Technology \\ ${ }^{2}$ Shaanxi University of Science and Technology Xi'an Campus
}

July 15, 2020

\begin{abstract}
As a high-precision measuring instrument, laser tracker is widely used in the field of geometric error detection of CNC machine tools. However, the employment of this laser tracker will lead to high cost as well as low measurement accuracy caused by the angle error. In order to solve these problems, the passive 3D laser tracking ball bar based on the principle of laser interference is introduced in this paper. The following measurement is realized by the passive stretching of the telescopic mechanism, and the space attitude adjustment of the laser is ensured by two precise rotating shafts. Moreover, the deflection caused by the telescopic guideway is an important factor affecting the accuracy of the device. Therefore, the telescopic mechanism is designed by the maximum deviation of the laser obtained by the experiment, and the finite element analysis is carried out. The results showed that the accuracy requirements are met. The main error model of the device is established and the influence of each error is analyzed. Moreover, the simulation results showed that the vertical axis offset angle error has the greatest impact on the device. At last, the reason of different influence of errors on the device is analyzed.
\end{abstract}

\section{Hosted file}

Development and Error Analysis of 3D Laser Tracking Ball Bar.pdf available at https: //authorea.com/users/343151/articles/469799-development-and-error-analysis-of-3d-lasertracking-ball-bar 